\title{
Item Profile Analysis for Tests Developed According to a Table of Specifications
}

\author{
Michael J. Kolen and David Jarjoura \\ The American College Testing Program
}

An approach to analyzing items is described that emphasizes the heterogeneous nature of many achievement and professional certification tests. The approach focuses on the categories of a table of specifications, which often serves as a blueprint for constructing such tests. The approach is characterized by profile comparisons of observed and expected correlations of item scores with category scores. A multivariate generaliza- bility theory model provides the foundation for the approach, and the concept of a profile of expected correlations is derived from the model. Data from a professional certification testing program are used for illustration and an attempt is made to provide links with test development issues and generalizability theory.

Many educational achievement and professional certification tests are heterogeneous in content, skills, or competencies measured. Tables of specifications that contain various content, skill, or competency categories are often used in the development of such tests to ensure content validity and to provide a framework for constructing multiple test forms. The variance components model for tests developed according to a table of specifications described by Jarjoura and Brennan $(1982,1983)$ is used in the present paper as a basis for an approach to analyzing individual items from such tests. The approach is illustrated using data from a professional certification test.

The approach to analyzing items described in the present paper explicitly considers the table of specifications. The major component is a profile of correlations between scores on an item and observed scores on each category of the table of specifications. The profile of correlations for an item from a particular category is compared to a similar profile of expected correlations for items from that same category. The approach focuses on a comparison of profile shapes rather than on the magnitude of any given correlation. The comparison of profile shapes is used to judge how typical or atypical a particular item is in comparison to other items from the same category.

This paper emphasizes the use of item profiles to study the functioning of individual test items in relation to the table of specifications. The profiles are used to generate hypotheses about items with atypical profiles, and the hypotheses are then evaluated by inspecting the content of the items. In this vein, the use of item statistics as the sole basis for selecting items is discouraged. 


\section{Table of Specifications Model}

In the model described by Jarjoura and Brennan $(1982,1983)$, the categories of the table of specifications are considered fixed conditions of measurement that characterize all test forms developed from the same table. Thus, each category of items is considered as a separate domain or universe. The category universe score for an examinee is defined as that examinee's expected proportion-correct score over samples of items within a category.

The number of categories in the table of specifications is denoted by $C$, and $c$ is used to index the categories. Letting $K_{c}$ be the number of items in category $c$, the total number of items is $K_{+}=$ $\sum_{c=1}^{c} K_{c}$. The categories are considered to be a fixed dimension and examinees and items-within-categories are random dimensions in the model. Scores on collections of items are always presented in the proportioncorrect metric. The potential item-level observations are

$Y_{p c k}=\mu_{c}+\pi_{p c}+\mathbf{l}_{k: c}+\xi_{p k: c}$

$p=1,2, \ldots, P ; c=1,2, \ldots, C ; k=1,2, \ldots, K_{c}$.

In this equation, $Y_{p c k}$ is the random variable representing the response of the $p$ th examinee to the $k$ th item of category $c$, and $\mu_{c}$ is a fixed category mean for the universe of items associated with category $c$ and the population of examinees. The other effects are random and defined to have expectations of zero. The $\pi_{p c}$ represent universe score effects, the $\iota_{k: c}$ represent item effects, and the $\xi_{p k: c}$ represent residuals composed of such effects as examinee-item interaction and response errors. A category universe score for an examinee is defined as $\mu_{c}+\pi_{p c}$. The colon $(k: c, p k: c)$ is used to indicate that $k$ is nested within $c$. For each of the three types of random effects, there are $C$ variance components-one for each fixed category. The variance components of interest are defined as expectations-over the population of examinees and universe of items in a category-of squares of these random effects. Covariances between universe scores in different categories are similarly defined. It is assumed that expectations of the random effects taken over the process that generates the observations described in Equation 1 are zero. Also, it is assumed that expectations of certain products and squares over this process give the components of interest.

Specifically, for the universe score effects,

$E \pi_{p c} \pi_{p c^{\prime}}=\sigma(\pi)_{c c^{\prime}} \quad c, c^{\prime}=1, \ldots, C$,

where $E$ denotes expectation. With $c=c^{\prime}$, this equation gives universe score variance for category $c$, and with $c \neq c^{\prime}$, it gives the covariance between universe scores for categories $c$ and $c^{\prime}$. These variance and covariance components will sometimes be referred to by the matrix $\Sigma$. Further, it is assumed that all effects in Equation 1, except for the $\pi_{p c}$ and $\pi_{p c^{\prime}}$, are uncorrelated.

For item effects,

$E \iota_{k: c}^{2}=\sigma^{2}(\iota)_{c} \quad c=1, \ldots, C$.

Finally, for the residual effects,

$E \xi_{p k: c}^{2}=\sigma^{2}(\xi)_{c} \quad c=1, \ldots, C$.

The expectations in Equations 2, 3, and 4 are not assumed to hold conditional on a particular sample of items or on a particular sample of examinees. The model itself and the motivation behind it are discussed in more detail by Jarjoura and Brennan (1982), and issues more relevant to generalizability theory are discussed by Jarjoura and Brennan (1983). Conditions that are sufficient for these assumptions are simple random sampling of examinees and items-within-categories (with samples selected independently) as well as uncorrelated response errors. Estimates of variance and covariance components and matrices are represented using a " ",' as in $\hat{\sigma}(\pi)_{c c}$. Jarjoura and Brennan $(1982,1983)$ suggested that the parameters 
of the model be estimated separately for each of a number of test forms generated from the same table of specifications and then averaged.

\section{Expected Correlations}

The concept of expected correlation is central to the approach presented in this paper. Consider an item from category $c$ and an observed score on category $c^{\prime}$ containing $K_{c^{\prime}}$ items. Conceptually, the expected correlation between an item from category $c$ and observed score on category $c^{\prime}$ is the average correlation between items randomly drawn from category $c$ and observed scores on sets of $K_{c^{\prime}}$ items randomly drawn from among potential category $c^{\prime}$ items. Note that if $c=c^{\prime}$, the item itself is not to be included in the observed score for category $c$.

The correlation between an item score and a category score is calculated as

$$
\hat{\boldsymbol{\rho}}\left(Y_{p c k}, Y_{p c^{\prime}}\right)=\frac{\sum_{p}\left(Y_{p c k}-Y_{c k}\right)\left(Y_{p c^{\prime} .}-Y_{c^{\prime}}\right)}{\left[\sum_{p}\left(Y_{p c k}-Y_{c k}\right)^{2} \sum_{p}\left(Y_{p c^{\prime} \cdot}-Y_{c^{\prime}}\right)^{2}\right]^{1 / 2}},
$$

where ".," denotes a mean. From the model above, the numerator and denominator have the following expected values:

$$
\begin{aligned}
& E \sum_{p}\left(Y_{p c k}-Y_{\cdot c k}\right)\left(Y_{p c^{\prime} \cdot}-Y_{\cdot c^{\prime}}\right)=(P-1) \sigma(\pi)_{c c^{\prime}}, \\
& E \sum_{p}\left(Y_{p c k}-Y_{\cdot c k}\right)^{2}=(P-1)\left[\sigma(\pi)_{c c}+\sigma^{2}(\xi)_{c}\right], \\
& \text { and } \\
& E \sum_{p}\left(Y_{p c^{\prime} \cdot}-Y_{c^{\prime} \cdot}\right)^{2}=(P-1)\left[\sigma(\pi)_{c^{\prime} c^{\prime}}+\sigma^{2}(\xi)_{c^{\prime}} / K_{c^{\prime}}\right] .
\end{aligned}
$$

Therefore,

$$
E \hat{\rho}\left(Y_{p c k}, Y_{p c^{\prime}}\right) \approx \frac{\sigma(\pi)_{c c^{\prime}}}{\left[\sigma(\pi)_{c c}+\sigma^{2}(\xi)_{c}\right]^{1 / 2}\left[\sigma(\pi)_{c^{\prime} c^{\prime}}+\sigma^{2}(\xi)_{c^{\prime}} / K_{c^{\prime}}\right]^{1 / 2}}
$$

is used for the expected correlation between an item score and a category score. Although the expected value of a function of random variables is not, in general, equal to the function of expected values, this is the usual approximation used (i.e., the first term of a Taylor series). (See Rajaratnam, Cronbach, \& Gleser, 1965, for a statistical discussion of a similar ratio and Cronbach, Gleser, Nanda, \& Rajaratnam, 1972 , p. 288, for a discussion of the use of such ratios.) Equation 9 is appropriate when an item is correlated with scores in its own category (i.e., when $c=c^{\prime}$ ) as long as scores on that item are not included in the calculation of the category $c$ observed score.

\section{Method for Analyzing Items}

A major component of the approach in this paper is a comparison of an observed correlation profile for an item with an expected profile. Suppose the following is obtained: (1) the correlation of an item, say item $k^{*}$, from category $c$ with observed score over all items in category $c$ except for itself, and (2) the correlation of item $k^{*}$ with observed scores on the $C-1$ other categories. Doing so enables the construction of a profile of the $C$ correlations. Also, using the expected correlation methodology described previously, a profile of $C$ expected correlations of items from category $c$ can be constructed by replacing the parameters of Equation 9 with accurate estimates. 
As illustrated below, a comparison of observed with expected profiles can be used to judge the similarity of a particular item to a "typical" item from category $c$. If the profile for an item is very similar in shape to the expected profile, then the item is judged to be functioning in a manner similar to a typical item from category $c$.

Although the procedures just described have broad applicability, in many situations a test form consists of a set of previously used items (that have been judged to be acceptable) as well as a set of new items (whose acceptability has yet to be judged). In order to avoid the contamination of category scores by (possibly) unacceptable new items, the new items can be eliminated from the computation of the category scores. With slight modification, the procedures described above can still be used. First, the estimates of expected correlations from Equation 9 are based only on previously used items. Second, only previously used items on the current test form are used to calculate the category observed scores. Then, the observed correlation profiles are constructed and compared to the expected profiles. The illustrative example is based on a testing program of this type. The profiles for the new items are the focus of this example.

\section{Illustration}

A professional certification test containing 150 multiple-choice questions is used in this illustration. This test is part of a battery that is used in the certification process. The test is developed according to a table of specifications that contains eight competency categories that are intended to measure those competencies that are most important for practicing in the profession. This particular test was chosen for illustrative purposes because its content specifications are relatively well defined and distinct, and most competency categories contain a reasonable number of items.

A pool of previously used test items is available to test developers for constructing new forms of this test. Approximately two-thirds of the items on any test form have been used previously, and the remaining one-third are new items. The data used in this illustration are based on the responses of 4,813 examinees administered Form $\mathrm{A}$ and 4,670 examinees administered Form B. The two forms were administered one year apart.

\section{Table of Specifications Model}

The parameters of the model were estimated from used items, rather than from the combination of new and used items. The model parameters were estimated separately for Forms A and B and then averaged. The numbers of previously used items per category for each form are shown in Table 1 , where the Roman numerals I through VIII denote competency categories. The parameter estimates are shown in Table 2. The proportion correct means $\left(\bar{\mu}_{c}\right)$ in the table range from about .73 (Category 1 ) to .81 (Category VIII), suggesting that the test is relatively easy for these examinees.

Disattenuated correlations among category universe scores are useful for evaluating the statistical heterogeneity of the test. For the table of specifications model, $\rho\left(\pi_{p c}, \pi_{p c^{\prime}}\right)=\sigma(\pi)_{c c^{\prime}}\left[\sigma\left(\pi_{c}\right) \sigma(\pi)_{c^{\prime} c^{\prime}}\right]^{-1 / 2}$ represents such a correlation between universe scores for categories $c$ and $c^{\prime}$. The estimated variance components shown in Table 2 were used to estimate the disattenuated correlations shown in Table 3 . It appears from the general magnitude of these correlations that the test is heterogeneous.

\section{Correlation Profiles}

The profile for a new item in this illustration consists of correlations between scores on the new item and observed scores on each of the categories. The profile for an item from a particular category is 
Table 1

Number of Previously Used Items Per Category

\begin{tabular}{cccccccccc}
\hline \hline & \multicolumn{10}{c}{ Form } & I & II & III & IV & V & VI & VII & VIII \\
\hline A & 26 & 18 & 16 & 16 & 11 & 11 & 11 & 4 \\
B & 26 & 13 & 15 & 10 & 11 & 10 & 10 & 4 \\
\hline
\end{tabular}

Table 2

Estimates of Variance and Covariance Components and Category Means ${ }^{a}$

\begin{tabular}{|c|c|c|c|c|c|c|c|c|}
\hline & \multicolumn{8}{|c|}{ Category } \\
\hline & $I$ & II & III & IV & $\mathrm{V}$ & VI & VII & VIII \\
\hline $\bar{\Sigma}$ & .0096 & & & & & & & \\
\hline & .0074 & .0078 & & & & & & \\
\hline & .0080 & .0083 & .0095 & & & & & \\
\hline & .0094 & .0085 & .0101 & .0117 & & & & \\
\hline & .0071 & .0062 & .0074 & .0074 & .0065 & & & \\
\hline & .0081 & .0075 & .0091 & .0106 & .0070 & .0098 & & \\
\hline & .0074 & .0077 & .0094 & .0094 & .0069 & .0082 & .0090 & \\
\hline & .0065 & .0046 & .0049 & .0055 & .0054 & .0046 & .0053 & .0052 \\
\hline $\bar{\sigma}^{2}(1){ }_{c}$ & .0195 & .0135 & .0168 & .0056 & .0212 & .0153 & .0086 & .0156 \\
\hline $\bar{\sigma}^{2}(\pi r)_{c}$ & .1685 & .1506 & .1481 & .1659 & .1672 & .1499 & .1387 & .1382 \\
\hline $\bar{\mu}_{c}$ & .7271 & .7804 & .7733 & .7594 & .7380 & .7765 & .8059 & .8078 \\
\hline
\end{tabular}

a $\bar{\Sigma}$ is used to denote the average of the estimates $(\hat{\Sigma})$ across the two forms. A similar notation is used to denote the other estimates.

Table 3

Disattenuated Correlations Among Content Category Scores

\begin{tabular}{rrrrrrrr}
\hline \multirow{2}{*}{ Category } & \multicolumn{7}{c}{ Category } \\
\cline { 2 - 7 } & I & II & III & IV & V & VI & VII \\
\hline II & .85 & & & & & & \\
III & .84 & .97 & & & & & \\
IV & .89 & .89 & .96 & & & & \\
V & .90 & .87 & .95 & .85 & & & \\
VI & .83 & .86 & .95 & .99 & .88 & & \\
VII & .80 & .91 & 1.01 & .92 & .90 & .88 & \\
VIII & .92 & .72 & .70 & .70 & .92 & .64 & .77 \\
\hline
\end{tabular}


compared to the profile of estimates of expected correlations of an item from that category (Equation 9). The magnitude of the expected correlation between an item and category score is mainly a function of the correlation between universe scores, such as those shown in Table 3 (equal to 1.0 if the item is in the same category as the category score), and the number of items contributing to the category score.

A profile of correlations was constructed for each new item on Form B. The numbers of new items per category on Form B are shown in Table 4. Only four of the items from Category I and four of the items from Category VI are discussed in detail. These items were chosen because they illustrate the present approach very well. Relevant data on the other items are included in Table 5, where items are ordered within category by their correlation with total score based on used items across categories on Form B. For example, Item 1 of Category $I$ is the item with the largest item-total correlation among Category I items.

The profiles of correlations for four Category I items are shown in Figure 1. The observed correlations are represented by triangles and the expected correlations by squares. Note that the expected profile is repeated in each of the graphs. From this figure, the Item 1 profile is generally higher than expected, the profiles for Items 2 and 3 are about as high as expected, and the Item 4 profile is lower than expected. More important than the general magnitude of correlations, however, is their pattern. The Item 1 profile is much more sharply peaked at Category I than is the expected profile. This suggests that Item 1 is more closely associated with the component that Category I items have uniquely in common than is the typical Category I item. In other words, there is some component common to Category I items that, on the average, sets them apart from items in other categories. When an item from category $c$ has a higher peak in its profile than the expected profile for any category, as the peak of Item 1 does for Category I, that item is referred to as being more associated with that category than is typical of items in category $c$.

Notice that the Item 2 profile is very similar to that of the typical Category I item. The pattern for Item 3 does not look like a Category I profile at all. This item seems to be more closely associated with Category III or Category VI than is typical of Category I items. Items 2 and 3 have very similar itemtotal correlations (.20 and .19 , respectively). Thus, two items from the same category with very similar item-total correlations can function very differently with respect to the table of specifications. Item 4 also has a somewhat discrepant pattern and appears to be more associated with Categories V or VIII than with its own category. Note also that the correlations of Item 4 with Categories V and VIII are very similar to the expected correlations for items from Categories V and VIII, respectively (see Table 5).

A major use of item statistics in this testing program is to flag items for review. The profiles suggest that Items 3 and 4 should be reviewed. Study of their content could reveal why they appear to be more associated with competencies other than their own.

Inspection of items with profiles like Item 1 may lead to a better understanding of the Category I competency. As this understanding and the testing program evolve, changes in the table of specifications may be desirable. These changes may be facilitated by the information gathered from the profiles. However, for current test forms, there is no reason to prefer an item like Item 1 over typical items. In this testing

Table 4

Number of New Items Per Category in Form B

\begin{tabular}{|c|c|c|c|c|c|c|c|}
\hline \multicolumn{8}{|c|}{ Category } \\
\hline$\vec{I}$ & II & $\overrightarrow{\text { III }}$ & IV & $\mathrm{V}$ & VI & VII & VIII \\
\hline 10 & 10 & 9 & 6 & 5 & 4 & 5 & 2 \\
\hline
\end{tabular}


Figure 1

Profiles of Correlation Between Item and Category Scores for Four Category I Items

(Expected Correlations are Represented by Squares, and Observed Correlations are Represented by Triangles)
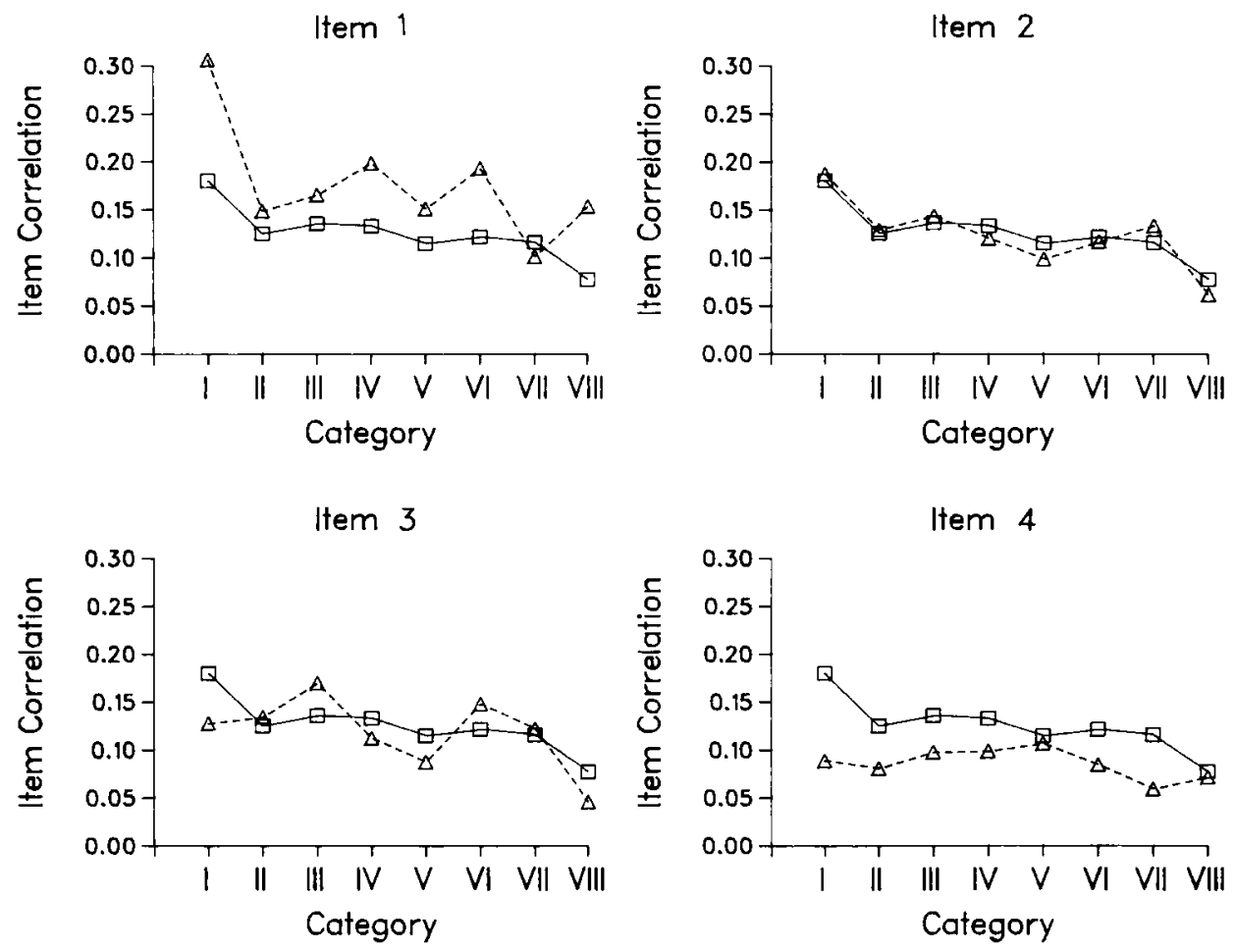

program only a total score is reported. If specific content scores were to be reported, it might then be desirable to construct test forms with greater differentiation among categories than current tests. If so, then the Category I specifications could be redefined to include items that would be more likely to have profiles similar to that of Item 1 .

Figure 2 contains profiles for the four new items from Category VI. The highest expected correlation in Figure 2 is for Category III. This results, in part, because Category III contains a greater number of used items than does Category VI, and Category III is relatively closely related to Category VI (disattenuated correlation of .95 from Table 3 ). This observation makes it clear that it is important to compare observed profiles to expected profiles in the current approach rather than to attempt an interpretation of the observed profiles in isolation.

Although Item 1 of Category VI tends to have the largest overall observed correlations, a comparison of its profile with the expected profile suggests that this item may be associated more with Category I than with its own category. The Item 3 profile is similar in shape to that for Item 1. Item 2 in Figure 2 is more associated with Category VI than the typical Category VI item and could provide greater differentiation from other categories. Item 4 has a pattern very similar to the typical Category VI item, although its correlations with the other categories are somewhat lower than is typical. 


\section{Figure 2}

Profiles of Correlation Between Item and Category Scores for Four Category VI Items

(Expected Correlations are Represented by Squares, and Observed Correlations are Represented by Triangles)
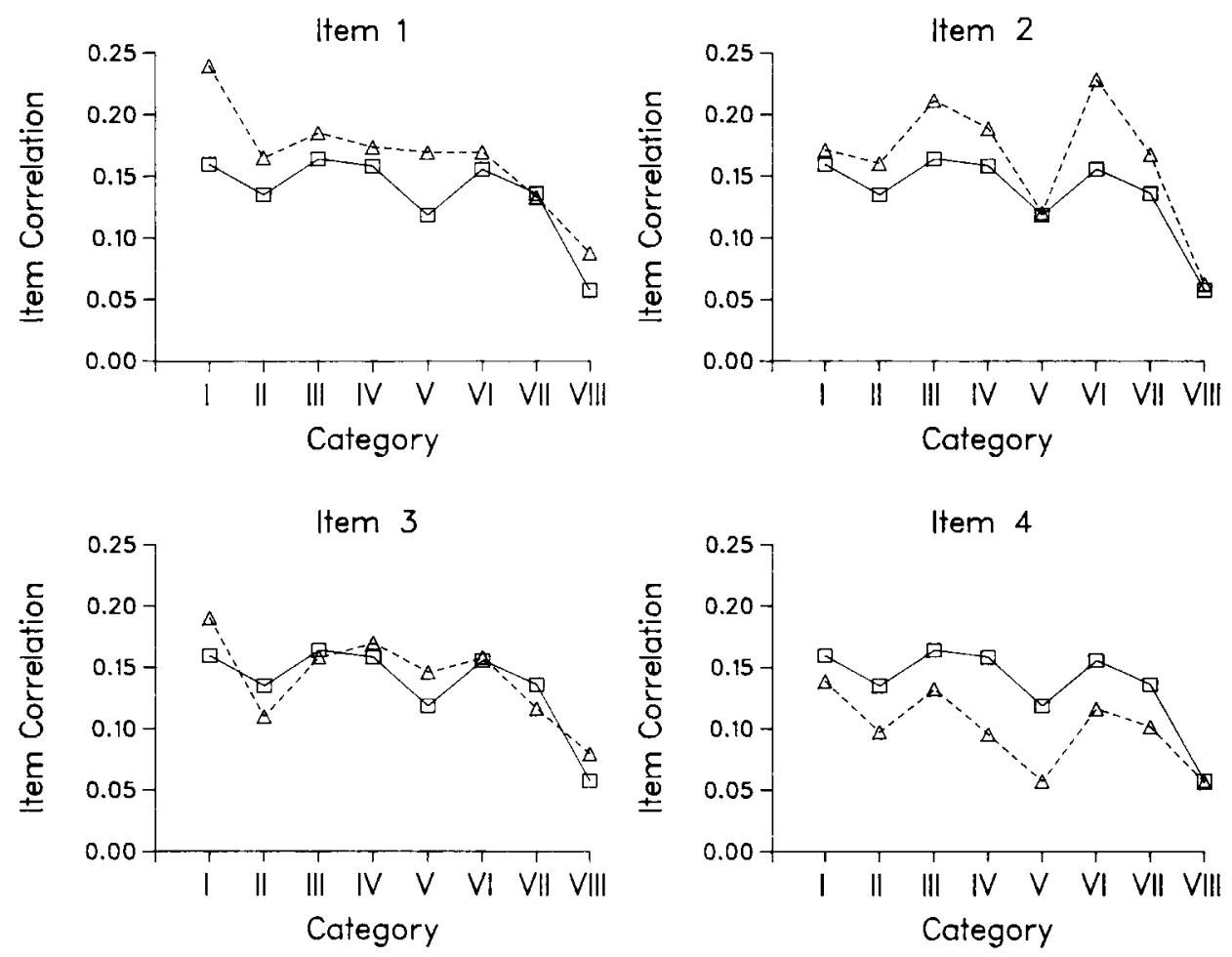

The profiles shown in Figure 2 suggest that Item 1 and possibly Item 3 should be reviewed. Also, study of Item 2 might lead to a better understanding of Category VI, and items like Item 2 might be desirable if the specifications for the test were redefined.

\section{Inspection of Item Content}

From an inspection of the content of the items whose profiles are shown in Figures 1 and 2, it appeared that Item 1 from Category I and Item 2 from Category VI strictly adhered to their respective competency statements. Item 3 from Category I and Items 1 and 3 from Category VI appeared to be correctly classified according to competency, but their content indicated that skills beyond their respective competencies were also being measured. The remaining items (Items 2 and 4 from Category I and Item 4 from Category VI) appeared to be correctly classified and did not seem to measure other skills to any substantial degree. In general, the review of content suggested that there was some degree of correspondence between profile similarity and content. More importantly, dissimilarity of observed and expected 
Table 5

Expected and observed Item Correlations with Category and Total Scores as well as Item Difficulty for New Form B Items

\begin{tabular}{|c|c|c|c|c|c|c|c|c|c|c|c|}
\hline \multirow[b]{2}{*}{ Category } & \multirow[b]{2}{*}{ I tem } & \multicolumn{6}{|c|}{ Correlation with Category } & \multicolumn{4}{|c|}{$\begin{array}{c}\text { Correlation } \\
\text { with }\end{array}$} \\
\hline & & I & II & III & IV & $\mathrm{V}$ & VI & VII & $\overline{V \bar{I}} \overline{\mathrm{I} I}$ & Total & Difficulty \\
\hline \multirow[t]{11}{*}{ I } & Exp. & .180 & .125 & .136 & .133 & .115 & .122 & .116 & .077 & .201 & .727 \\
\hline & 1 & .306 & .149 & .166 & .199 & .151 & .193 & .101 & .153 & .284 & .834 \\
\hline & 2 & .187 & .129 & .143 & .120 & .098 & .117 & .134 & .061 & .199 & .928 \\
\hline & 3 & .128 & .134 & .170 & .112 & .087 & .148 & .122 & .046 & .186 & .625 \\
\hline & 4 & .089 & .080 & .098 & .099 & .107 & .084 & .059 & .071 & .129 & .914 \\
\hline & 5 & .100 & .077 & .097 & .095 & .087 & .091 & .060 & .031 & .127 & .960 \\
\hline & 6 & .082 & .106 & .094 & .091 & .106 & .078 & .050 & .044 & .125 & .988 \\
\hline & 7 & .082 & .082 & .089 & .079 & .110 & .077 & .056 & .064 & .120 & .986 \\
\hline & 8 & .088 & .096 & .092 & .045 & .050 & .083 & .077 & .023 & .111 & .853 \\
\hline & 9 & .082 & .070 & .081 & .034 & .048 & .033 & .060 & .037 & .093 & .682 \\
\hline & 10 & .017 & .038 & .004 & .007 & -.007 & .046 & .009 & .000 & .023 & .659 \\
\hline \multirow[t]{11}{*}{ I I } & Exp. & .146 & .141 & .150 & .127 & .106 & .120 & .127 & .058 & .193 & .780 \\
\hline & 1 & .186 & 192 & .145 & .121 & .107 & .120 & .149 & .045 & .215 & .889 \\
\hline & 2 & .167 & .151 & .133 & .106 & .146 & .095 & .104 & .065 & .193 & .969 \\
\hline & 3 & .172 & .110 & .117 & .102 & .108 & .074 & .074 & .084 & .169 & .947 \\
\hline & 4 & .132 & .118 & .103 & .083 & .044 & .104 & .113 & .031 & .148 & .928 \\
\hline & 5 & .063 & .100 & .079 & .110 & .096 & .121 & .098 & .054 & .131 & .991 \\
\hline & 6 & .098 & .095 & .073 & .066 & .091 & .086 & .082 & .032 & .122 & .989 \\
\hline & 7 & .092 & .091 & .090 & .067 & .054 & .079 & .069 & .026 & .114 & .294 \\
\hline & 8 & .092 & .079 & .064 & .065 & .089 & .066 & .081 & .053 & .113 & .996 \\
\hline & 9 & .057 & .075 & .052 & .069 & .066 & .050 & .067 & .018 & .087 & 990 \\
\hline & 10 & .039 & .027 & .017 & .055 & .017 & .045 & -.001 & .041 & .044 & .809 \\
\hline \multirow[t]{10}{*}{ III } & Exp. & .158 & .150 & .171 & .152 & .127 & .146 & .156 & .062 & .220 & .773 \\
\hline & 1 & .181 & .205 & .251 & .270 & .145 & .224 & .222 & .057 & .301 & .620 \\
\hline & 2 & .182 & .173 & .229 & .240 & .112 & .210 & .164 & .051 & .268 & .797 \\
\hline & 3 & .152 & .147 & .163 & .136 & .152 & .118 & .130 & .106 & .211 & .895 \\
\hline & 4 & .136 & .129 & .156 & .161 & .123 & .138 & .093 & .055 & .193 & .966 \\
\hline & 5 & .146 & .136 & .147 & .137 & .130 & .110 & .109 & .066 & .192 & .734 \\
\hline & 6 & .129 & .112 & .171 & .138 & .129 & .129 & .105 & .047 & .188 & .345 \\
\hline & 7 & .107 & .129 & .178 & .138 & .100 & .100 & .128 & .051 & .181 & .420 \\
\hline & 8 & .081 & .215 & .129 & .118 & .104 & .100 & .092 & .018 & .147 & .754 \\
\hline & 9 & .053 & .062 & .051 & .054 & .057 & .047 & .048 & .037 & .077 & .745 \\
\hline \multirow[t]{7}{*}{ IV } & Exp. & .177 & .145 & .173 & .165 & .119 & .160 & .147 & .065 & .225 & .759 \\
\hline & 1 & .163 & .188 & .202 & .183 & .143 & .173 & .161 & .064 & .249 & .821 \\
\hline & 2 & .167 & .207 & .182 & .159 & .138 & .122 & .154 & .064 & .235 & .837 \\
\hline & 3 & .149 & .152 & .168 & .159 & .113 & .186 & .154 & .049 & .219 & .749 \\
\hline & 4 & .112 & .166 & .167 & .170 & .081 & .130 & .117 & .023 & .190 & .653 \\
\hline & 5 & .114 & .102 & .176 & .128 & .112 & .139 & .122 & .028 & .181 & .672 \\
\hline & 6 & .120 & .108 & .135 & .117 & .076 & .090 & .121 & .051 & .161 & .912 \\
\hline \multirow[t]{6}{*}{ v } & Exp. & .135 & .107 & .128 & .105 & .106 & .106 & .109 & .064 & .167 & .738 \\
\hline & 1 & .216 & .200 & .250 & .204 & .196 & .207 & .202 & .083 & .304 & .838 \\
\hline & 2 & .166 & .178 & .255 & .220 & .151 & .218 & .172 & .023 & .273 & .750 \\
\hline & 3 & .154 & .170 & .200 & .170 & .167 & .133 & .152 & .099 & .238 & .742 \\
\hline & 4 & .098 & .060 & .070 & .066 & .094 & .049 & .050 & .068 & .108 & .811 \\
\hline & 5 & .070 & .071 & .066 & .050 & .035 & .044 & .069 & .032 & .086 & .687 \\
\hline \multirow[t]{5}{*}{ VI } & Exp. & .159 & .135 & .164 & .158 & .118 & .155 & .136 & .057 & .212 & .777 \\
\hline & 1 & .239 & .164 & .185 & .273 & .169 & .169 & .133 & .087 & .264 & .871 \\
\hline & 2 & .171 & .160 & .211 & .188 & .120 & .228 & .167 & .062 & .254 & .929 \\
\hline & 3 & .190 & .110 & .258 & .170 & .146 & .158 & .116 & .079 & .222 & .908 \\
\hline & 4 & .138 & .097 & .132 & .095 & .057 & .116 & .101 & .056 & .158 & .968 \\
\hline \multirow[t]{6}{*}{ VII } & Exp. & .152 & .143 & .175 & .145 & .122 & .136 & .155 & .069 & .220 & .806 \\
\hline & 1 & .276 & .202 & .228 & .210 & .174 & .204 & .234 & .097 & .322 & .766 \\
\hline & 2 & .221 & .204 & .233 & .208 & .161 & .182 & .231 & .110 & .301 & .672 \\
\hline & 3 & .127 & .129 & .138 & .096 & .134 & .097 & .167 & .101 & .186 & .870 \\
\hline & 4 & .069 & .080 & .096 & .050 & .041 & .030 & .085 & .003 & .094 & .781 \\
\hline & 5 & .050 & .032 & .036 & .020 & .048 & .044 & .059 & .012 & .059 & .986 \\
\hline VIII & Exp. & .135 & .087 & .094 & .086 & .096 & .077 & .092 & .069 & .145 & .808 \\
\hline & 1 & .078 & .090 & .099 & .088 & .067 & .096 & .126 & .041 & .130 & .928 \\
\hline & 2 & .056 & .122 & .107 & .070 & .066 & .052 & .128 & .062 & .122 & .870 \\
\hline
\end{tabular}


profiles usually indicated that an item did not adhere strictly to competency statements. The review of items improved understanding of the functioning of the table of specifications.

\section{Discussion}

The item profile analysis described can be used most advantageously as one component of an item analysis strategy in which item statistics are used to flag items for review. Using such a strategy, a flagged item is removed from the test or item pool only after its content is found to be problematic. This strategy may be most appropriate for educational achievement tests with well defined tables of specifications and for which test content is of the utmost concern. The item profile analyses might be less appropriate for item analysis strategies that use item statistics as a direct basis for item selection.

In the illustrative example, very large sample sizes of examinees were used, most of the categories of the table of specifications contained relatively large numbers of items, and the categories were relatively distinct and well defined. These test characteristics probably are optimal for using the item profile procedures, and further research should be conducted to assess the usefulness of the technique under less optimal circumstances.

In the illustrative example, visual inspection of the profiles was used to decide when an item had a profile that was atypical. Even though such a procedure produced useful data for interpretation in the illustrative example, it might be desirable to have a more precise way for deciding when an item has a profile that is atypical. A procedure that takes into account sampling error variances and covariances in estimating the correlations that comprise the profile could be developed to provide a sampling error baseline for judging the degree to which the profile for an item is atypical. The development of such a procedure would be quite complex and for this reason should be considered as an area for future research.

The approach to analyzing items described in this paper stresses heterogeneity in terms of content, skills, or competencies measured. The statistical treatment required in this approach is more complicated than standard treatments that use item-total correlations (item discrimination indices). However, the additional complexity is necessary to avoid many of the problems associated with using standard item discrimination indices. When standard indices are used in the selection of items or in flagging items for review, they (1) may favor items from categories containing larger numbers of items, (2) can lead to greater test homogeneity than originally intended (Henrysson, 1971, p. 135), (3) can lead to an emphasis on global scores at the expense of the interpretability of specific content scores (Linn, 1980, p. 88), and (4) tend not to identify items that are ambiguously classified according to content. The methods for analyzing items described in this paper can be used in addition to the standard statistics to help avoid these problems. In addition, the procedure described here provides valuable information about the functioning of the table of specifications, whereas the usual item discrimination indices do not.

\section{Conclusion}

According to Brennan (1983) "In treatments of classical test theory, the subject of item analysis is generally discussed as a separate topic with weak links to the classical model and associated concepts of reliability and validity. . . . A similar statement applies with about equal force to generalizability theory" (p. 123). The present paper is an attempt to provide a link between item analysis and generalizability theory. Also, because this approach takes into account the heterogeneity of many educational tests, it provides for a stronger link between test development, item analysis, and psychometric theory than other approaches for such tests. 


\section{References}

Brennan, R. L. (1983). Elements of generalizability theory. Iowa City IA: The American College Testing Program.

Cronbach, L. J., Gleser, G. C., Nanda, H., \& Rajaratnam, N. (1972). The dependability of behavioral measurements: Theory of generalizability for scores and profiles. New York: Wiley.

Henrysson, S. (1971). Gathering, analyzing, and using data on test items. In R. L. Thorndike (Ed.), Educational measurement (2nd ed.). Washington DC: American Council on Education.

Jarjoura, D., \& Brennan, R. L. (1982). A variance components model for measurement procedures associated with a table of specifications. Applied Psychological Measurement, 6, 161-171.

Jarjoura, D., \& Brennan, R. L. (1983). Multivariate generalizability models for tests developed from tables of specifications. In L. J. Fyans (Ed.), New directions for testing and measurement. Generalizability theory: Inferences and practical applications. San Francisco: Jossey-Bass.

Linn, R. L. (1980). Test design and analysis for measurement of educational achievement. In W. B. Schrader (Ed.), New directions for testing and measurement. Measuring achievement: Progress over a decade. San Francisco: Jossey-Bass.

Rajaratnam, N., Cronbach, L. J., \& Gleser, G. C. (1965). Generalizability of stratified-parallel tests. Psychometrika, 30, 39-56.

\section{Author's Address}

Send requests for reprints or further information to $\mathrm{Mi}$ chael J. Kolen, American College Testing Program, P.O. Box 168, Iowa City IA 52243, U.S.A. 